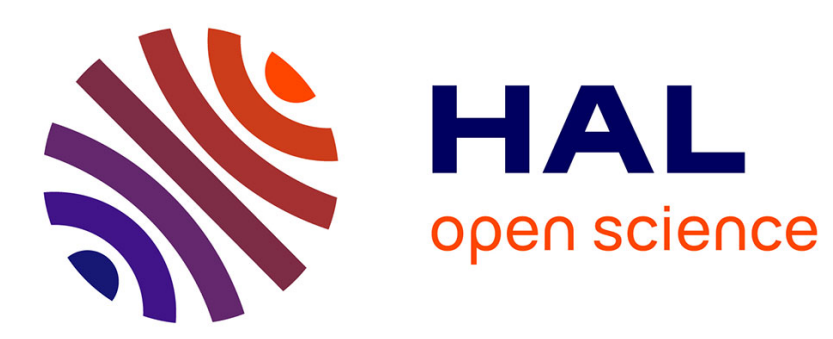

\title{
Signs of Timing in Motor Cortex During Movement Preparation and Cue Anticipation
}

Bjørg Elisabeth Kilavik, Joachim Confais, Alexa Riehle

\section{To cite this version:}

Bjørg Elisabeth Kilavik, Joachim Confais, Alexa Riehle. Signs of Timing in Motor Cortex During Movement Preparation and Cue Anticipation. Advances in Experimental Medicine and Biology, 2014, Neurobiology of Interval Timing, 829, pp.121-142. 10.1007/978-1-4939-1782-2_7 . hal-01464159

\section{HAL Id: hal-01464159 \\ https://hal-amu.archives-ouvertes.fr/hal-01464159}

Submitted on 16 Feb 2017

HAL is a multi-disciplinary open access archive for the deposit and dissemination of scientific research documents, whether they are published or not. The documents may come from teaching and research institutions in France or abroad, or from public or private research centers.
L'archive ouverte pluridisciplinaire HAL, est destinée au dépôt et à la diffusion de documents scientifiques de niveau recherche, publiés ou non, émanant des établissements d'enseignement et de recherche français ou étrangers, des laboratoires publics ou privés. 


\title{
Signs of Timing in Motor Cortex During Movement Preparation and Cue Anticipation
}

\author{
Bjørg Elisabeth Kilavik, Joachim Confais, and Alexa Riehle
}

\begin{abstract}
The capacity to accurately anticipate the timing of predictable events is essential for sensorimotor behavior. Motor cortex holds an established role in movement preparation and execution. In this chapter we review the different ways in which motor cortical activity is modulated by event timing in sensorimotor delay tasks. During movement preparation, both single neuron and population responses reflect the temporal constraints of the task. Anticipatory modulations prior to sensory cues are also observed in motor cortex when the cue timing is predictable. We propose that the motor cortical activity during cue anticipation and movement preparation is embedded in a timing network that facilitates sensorimotor processing. In this context, the pre-cue and post-cue activity may reflect a presetting mechanism, complementing processing during movement execution, while prohibiting premature responses in situations requiring delayed motor output.
\end{abstract}

\section{Keywords}

Motor cortex - Timing - Delay tasks - Movement preparation - Cue anticipation

\section{Introduction: Sensorimotor Delay Tasks}

When a tennis player tracks a ball in motion during a match, he precisely times when his hand should swing the racket to intercept the ball. Similarly, when a driver anticipates the traf-

B.E. Kilavik • J. Confais $(\bowtie) \cdot$ A. Riehle Institut de Neurosciences de la Timone (INT), CNRS Aix Marseille Université, Marseille, France e-mail: joachim.confais@gmail.com fic light turning green, he/she uses an internal representation of elapsed (or remaining) time before stepping on the gas pedal. These examples illustrate that past experience and environmental clues are used to accurately anticipate the timing of predictable events, thereby improving sensorimotor behavior. In the laboratory, pre-cued motor tasks (Fig. 1) are often used to study movement preparation processes. In such tasks, movements are initiated faster when the GO signal timing is known in advance ([1-4], see reviews in $[5,6])$. 


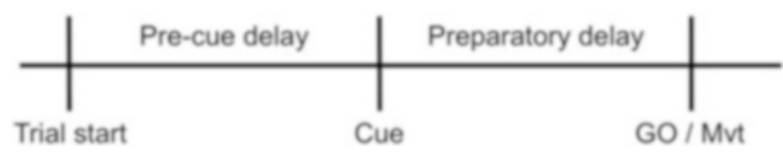

Fig. 1 A generic sensorimotor delay task. Instructed delay tasks typically use a (warning) cue followed after a delay by an imperative GO signal. The cue might provide full, partial or no information about the movement to be executed after GO. During the preparatory delay between the cue and GO, the movement can be prepared using the available information. Note that in some cases, there is not explicit GO signal, and movement onset (Mvt) should be timed by the subject (self-paced). Furthermore, there is often a pre-cue delay of predictable duration, whenever there is an initial external signal or selfgenerated movement (trial start) preceding the cue that provides temporal information. During the pre-cue delay, the subject can anticipate the moment of cue occurrence (temporal orienting) in order to optimize cue detection

More generally, when temporal uncertainty about the GO signal occurrence decreases, the behavioral reaction time (RT) also decreases. By introducing multiple delay durations presented at random from trial to trial, RT will significantly increase on average, as the notion of a predictable event is confounded. However, because in this case the conditional probability of receiving a GO signal increases with time, RT will decrease for increasing delay durations [1]. Furthermore, even when the delay duration is constant RTs vary from trial to trial, suggesting a variable representation of subjective delay duration across trials $[6,7]$.

The strong dependency of sensorimotor behavior on time estimation processes has motivated a growing interest to uncover their neural correlates. Niki and Watanabe [8] were the first to connect anticipatory single neuron delay activity to implicit time estimation. Since then, several studies interpreted neuronal discharge during delays as being related to timing processes, both in sensory and motor areas $[3,4$, 7, 9-26]. These studies used tasks in which an informative cue is followed by a delay prior to an imperative GO signal or a self-timed movement initiation (Fig. 1), implying either implicit or explicit timing processes, respectively [27]. In an instructed delay task the subject must process the cue during the preparatory delay and prepare the movement, whilst simultaneously avoiding premature movement release. The preparatory delay between the cue and the GO signal either has a fixed duration or varies from trial to trial between a minimal and maximal duration. The presence of a GO signal obviates the need to estimate delay duration explicitly in order to perform the task correctly. However, the fact that RTs are faster when the timing of GO is more predictable confirms that this timing information is implicitly exploited in order to optimize performance (e.g., receiving the reward sooner in the case of animal subjects). A different approach is needed to study explicit timing. Here the subject is asked to provide an estimate of the delay duration, either by self-timing a movement initiation (no final GO signal provided; e.g., [3, $21,22,25,28-30]$ ), tapping rhythmically (e.g., [31-33]; reviewed in the following chapter in this volume), intercepting a moving target (see review in [34]), associating a particular motor response with a particular delay duration [3], or comparing two delay durations (e.g., [13, 35, 36]).

Importantly, most sensorimotor delay tasks also contain a well-defined pre-cue epoch, with either a fixed or a variable (but predictable) duration (Fig. 1). The event that marks the start of this pre-cue delay can either be self-generated by the subject, such as pressing a start-button or ending the movement in the preceding trial, or it can be an external signal presented to the subject. In sensory cortex, it was shown that the pre-cue anticipatory activity in visual area V4 was modulated by the hazard rate of the visual cue [12]. Furthermore, anticipatory activity preceding somatosensory stimulation has been shown in somatosensory cortex [37, 38], and in auditory cortex preceding auditory stimuli [39]. However, such cue anticipatory neuronal activity is not restricted to sensory areas, as it can also be found in motor cortex [9, 10, 40, 41].

In this chapter we will examine the different ways in which timing affects neuronal activity in motor cortex. We will first show that task timing organizes motor cortical activity during movement preparation, observable in both the spiking activity of single neurons and in different neuronal population measures. Sensorimotor behavior 
is a distributed process, most likely with several areas working in parallel during different task epochs. In line with this idea, we will show that motor cortical activity is already modulated before movement preparation, e.g., during the anticipation of sensory cues.

We propose that the motor cortical activity during cue anticipation and movement preparation is embedded in a timing network that facilitates sensorimotor processing. In particular, activity prior to and following the sensory cue may reflect presetting mechanisms that complement the subsequent processing during movement execution, whilst prohibiting a premature response in situations requiring a delayed motor output.

\section{Part 1: Timing During Movement Preparation}

\section{Spiking Activity During Movement Preparation}

Neurons in many cortical and sub-cortical areas change their firing rate progressively during the preparatory delay $[3,4,8,9,13,16,24,25,35$, 42-46]. Such climbing activity can be observed in both self-paced, explicit timing tasks (see example in [47]) and implicit timing tasks using a GO signal (Fig. 2a, b). These neuronal activity patterns have been successfully used in single trials to decode the elapsed time of an event/ movement and the onset time of subsequent events/movements [13, 25, 26]. Climbing activity has also been tested as a timing mechanism in modeling approaches [48-50]. One approach proposed that task timing modulates the slope, but not the final peak of activity. Thus, a threshold mechanism could read-out the end of a timed interval [50].

Okamoto et al. [51] suggested that climbing activity observable in across-trial averages might instead be a result of variable transition times of bimodal activity. They described single neuron activity from anterior cingulate cortex with bimodal firing rate distributions and a large across-trial variability, matching well their proposed model. The generality of this proposition still remains to be explored in other brain areas where climbing activity was reported. In data recorded from primary motor cortex (M1) and dorsal premotor cortex (PMd) previously presented in Confais et al. [41], we calculated spike count distributions of neurons with climbing activity. None of the 58 selected neurons with climbing activity had bimodal spike count distributions during the preparatory delay (all selected neurons had a minimal firing rate of 10 spikes/s at GO, spike counts measured in $200 \mathrm{~ms}$ sliding windows with $100 \mathrm{~ms}$ overlap). This preliminary result suggests that bimodal activity patterns do not account for the climbing activity observed in trial averages during movement preparation in motor cortical areas.

However, if climbing activity reflects a timing mechanism, the slope of the firing rate should be modulated with delay duration, the slope being steeper in short than in long delays. Furthermore, the onset of the climbing activity should start at the beginning of the delay, possibly preceded by an initial phasic response to the cue. Thus, the time of onset should be independent of the duration. When exploring this in the aforementioned dataset of 58 neurons, we found that only three neurons had similar onset latencies, but different slopes in short and long delays. Several neurons displayed a change in slope combined with a change in onset latency (13/58; see example neuron in Fig. 2a), and the majority kept the slope constant with a pure shift in onset latency (41/ 58). This suggests that motor cortical climbing activity clearly reflects task timing, and can even be used to decode time ([25]; note that neurons with an onset difference will also be informative for delay duration), but does not seem to be a self-sufficient mechanism responsible for tracking time. Rather, this activity might be embedded in a context-dependent timing network.

As time estimation is at the core of anticipatory behavior it is reasonable to ask if neuronal delay activity correlates with the subjective estimate of time. As a consequence of the scalar property of time estimation processes [52], the variability in time estimation increases continuously as time passes during the delay. This scalar 
a The onset and slope of climbing activity scales to delay duration
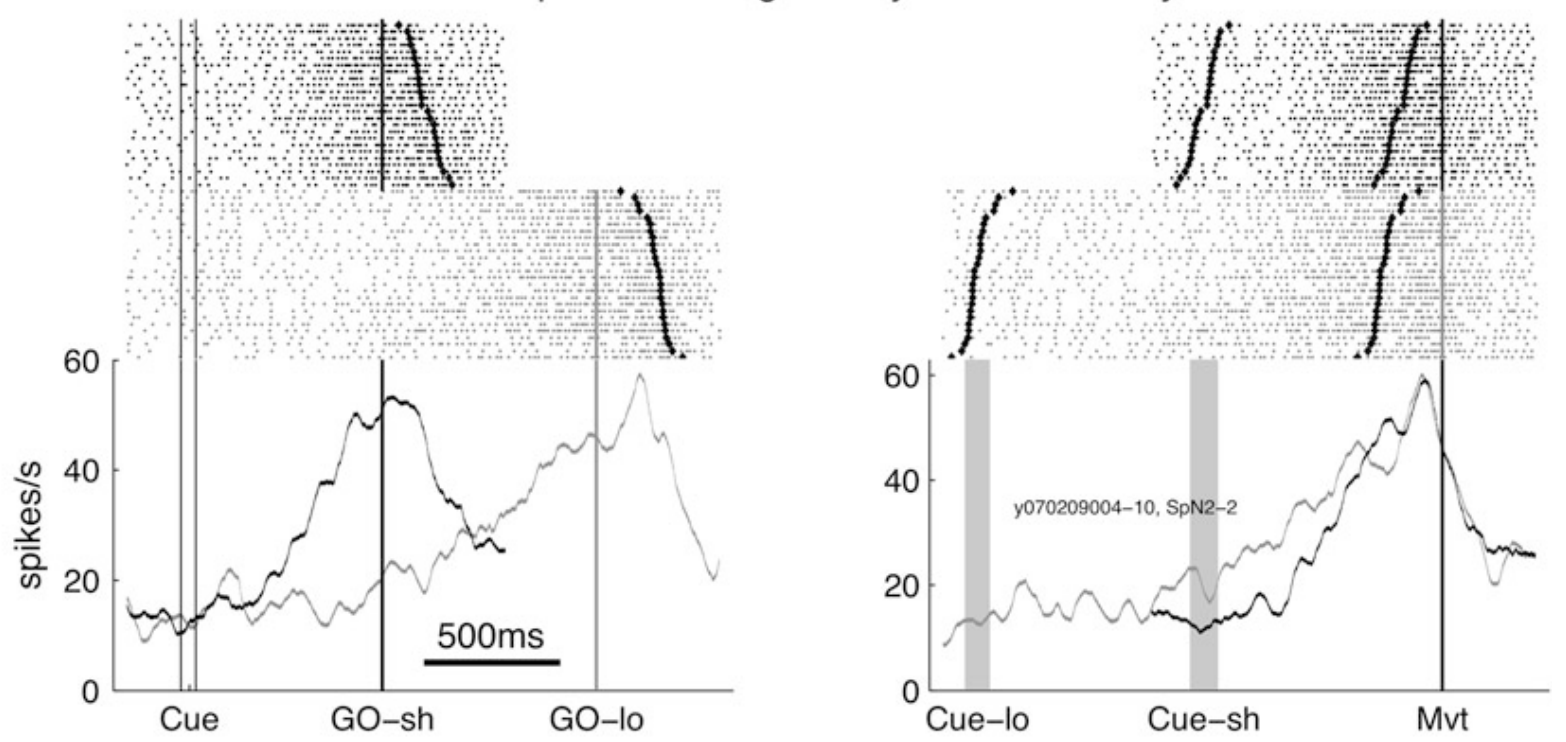

b

Spiking activity scales to internal timing, trial-by-trial

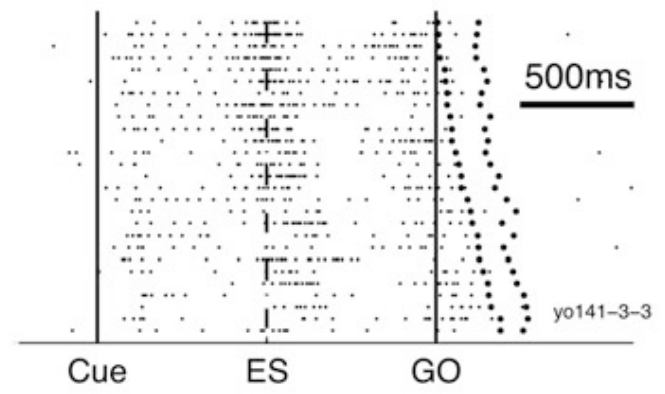

C

LFP beta power (monkey T)

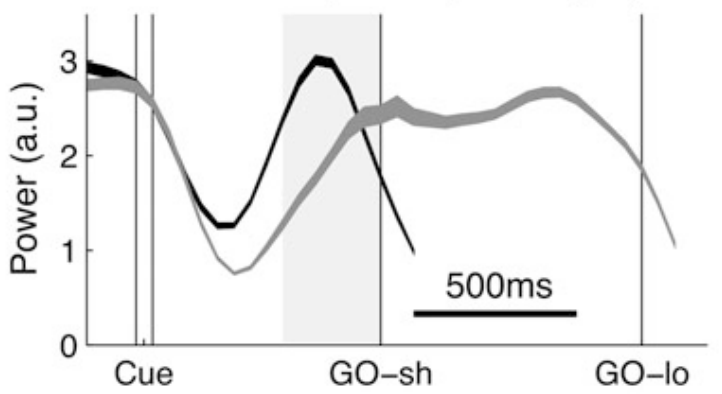

C LFP beta frequency (monkey T)

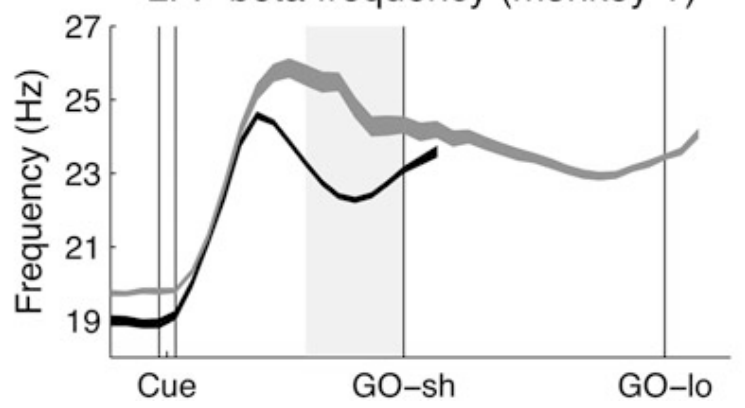

Fig. 2 Scaling of motor cortical activity during movement preparation. (a) Example of a neuron that adjusts (scales) both the onset and the slope of climbing activity

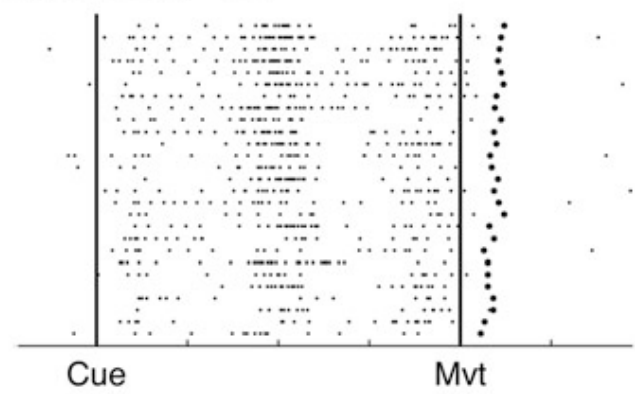

d

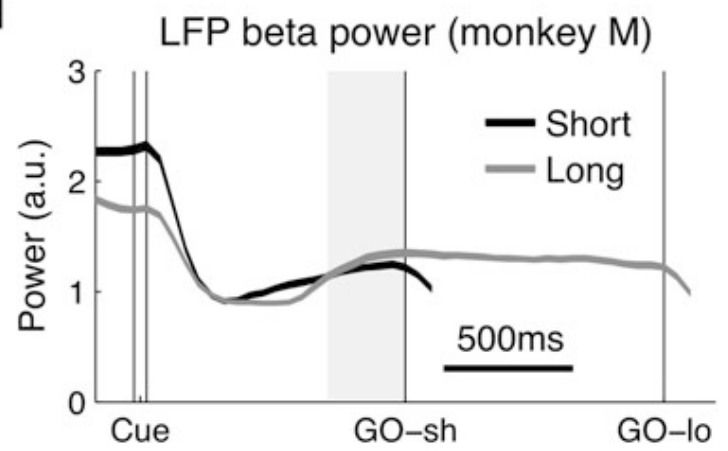

e
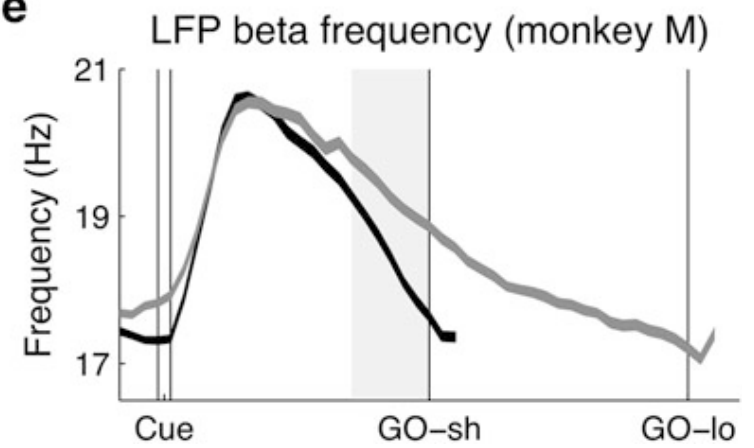

during movement preparation to the delay duration between the cue and GO. On top, the trial-by-trial activity is shown for short and long delay trials as raster plots, 
property may then be reflected in the increasing variability of neuronal delay activity. Renoult et al. [7] studied the influence of temporal prior information on neuronal delay activity in monkey motor cortex during a task in which two equally probable delay durations were randomly presented.

The neuron presented in Fig. 2b (left panel) showed three distinct epochs of increased activity during long delay trials: the first after presentation of the cue, the second around the expected GO signal (ES) at the end of the short delay (50\% probability), and the third towards the actual occurrence of the GO signal at the end of the long delay. The increased activity during the second and third epochs appeared without the presentation of any external signal, indicating a fairly accurate estimate of the delay durations. Whereas the activity increase following the cue was clearly time-locked to the stimulus, the activity during the third epoch was clearly aligned to movement onset, trial-by-trial. The activity during the second epoch around ES had an intermediate alignment. Thus, neuronal activity went from being aligned to the occurrence of an external signal (cue) to being aligned to movement onset via some intermediate alignment to an internal signal (ES), and across-trial variability in the temporal profile of neuronal discharge increased throughout the delay. Renoult et al. [7] hypothesized that if one considers the animal's subjective timing of the delay as the elapsed time between the cue and movement onset, then suppressing the temporal variability in RT should decrease the across-trial variability in neuronal discharge. Here the cue is considered as being t0, where time is reset in each trial. The time between the cue ( $t 0)$ and movement onset was kept identical across trials by first defining a new time scale per trial and then rescaling it across trials. Each spike was then displaced in time accordingly (i.e., the farther a spike from t0 the larger its displacement). As expected, the variability in the timing of neuronal peak discharges no longer increased during the trial (Fig. $2 b$ right panel). This suggests a
Fig. 2 (Continued) with individual lines for each behavioral trial and each dot representing an action potential. The peri-event time histograms (PETHs) are shown in the bottom panels. To the left, the data of short and long delay trials is aligned to cue onset. Clearly the onset of the increase in activity after the cue occurs later in long delay trials. To the right, the data is aligned to movement onset. Whereas the peak in activity shortly preceding movement onset is the same for short and long delay trials, the slope of the increase in activity is steeper for short delay trials. The gray rectangles represent the average $( \pm$ std) time of cue onset in long and short delay trials. The larger black dots in the raster plots represent movement onset (left plots) or cue onset (right plots). Trials are aligned offline according to increasing RT. The duration of the cue was $55 \mathrm{~ms}$, the short delay $700 \mathrm{~ms}$ and the long delay 1,500 ms. For more details on the task, see Confais et al. [41] (unpublished data from J Confais, BE Kilavik, A Ponce-Alvarez and A. Riehle). (b) Raster plots of an example neuron recorded during a task in which the GO signal could be presented either after a short or long delay duration. Here only long delay trials are shown, and ES (expected signal, left plot) represents the moment in which the animal expected the GO signal with $50 \%$ probability. To the left, trials are aligned to external signals. To the right, trials are re-scaled according to the duration between the cue and movement onset (Mvt). See the text and Renoult et al. [7] for more details on the task and analysis (unpublished data from: A. Riehle, L. Renoult and S. Roux). (c, d) Averaged normalized beta peak power between cue and GO ( \pm sem $)$ across 191 LFPs for monkey $\mathrm{T}$ and 631 for monkey $\mathrm{M}$, recorded in the same task as the example neurons shown in a. Monkey $\mathrm{T}$ (c) had a systematic difference between short and long delay trials, with a majority of LFPs having higher power in the end of short trials than in the middle of long trials (light gray window; same moment in time after the cue; $72 \%$ of LFPs with significantly higher power in short than in long trials, compared to only $10 \%$ with an opposite effect; 2-way ANOVA, movement direction and delay duration as factors). In monkey $\mathrm{M}$ (d), there was no systematic power difference between short and long trials across LFPs, but overall power changed only little during the preparatory delay. (e, f) Averaged beta peak frequency between cue and GO $( \pm$ sem $)$ for the same LFPs as shown in c, d. Many LFPs had significantly different beta peak frequency comparing the end of short trials with the middle of long trials (gray window). A majority had lower frequency in short trials (70 and $26 \%$ of LFPs in monkey $\mathrm{T}$ and $\mathrm{M}$, respectively; only $3 \%$ had an opposite effect). For both monkeys, cue duration was $55 \mathrm{~ms}$. For monkey $\mathrm{T}$, the short and long delays were 700 and $1,500 \mathrm{~ms}$, while they were 1,000 and $2,000 \mathrm{~ms}$ for monkey M. For more details of task and data analysis, please see Kilavik et al. [93] (c-f: unpublished data from Kilavik and Riehle; preliminary results presented in [47]) 
direct link between the temporal profile of spiking activity and time estimation. The timing of motor cortical activity reflects the elasticity ("rubberband") of the animal's subjective time.

Finally, Roesch and Olson [19] found that many of the neurons in frontal areas (including premotor cortex close to the arcuate sulcus) that were sensitive to delay duration, were also sensitive to reward magnitude in a saccade task. These neurons typically exhibited higher firing rate shortly after the presentation of a cue indicating a short delay or a large forthcoming reward. This result might, at least in part, be explained by the notion that waiting for a long delay before receiving a reward decreases the subjective value of the reward (time discounting; [53]), suggesting that reward and delay duration might both act on general motivation. Indeed, in monkey studies, effects related to reward and GO signal expectancy cannot be clearly dissociated from the effects related to timing in many cases (but see [26, 54]). However, climbing activity also occurs in the absence of immediate reward, reflected in population activity measures in human participants described below.

\section{Population Activity During Movement Preparation}

The extracellular local field potential (LFP) may be recorded from the same electrode as spikes, by low-pass filtering of the raw signal (e.g., below $250 \mathrm{~Hz}$ ), and is modulated in parallel to single neuron discharge. It is considered to mainly reflect the (sub-threshold) synaptic activity in a large population of neurons, with additional contributions from spike-after potentials or intrinsic trans-membrane current changes [55-57]. Since the LFP sums activity around the electrode, modulations observable in the LFP must reflect more or less synchronous activity in a sufficiently large population of neurons, possibly indicating a degree of coherent network activity $[58,59]$. Currently there is a great interest in understanding the relationship between the spiking activity of single neurons and the slower fluctuations of the LFP (e.g., [60, 61]). The intra- cerebral LFP is related to the externally recorded electro- and magneto-encephalographic (EEG/ MEG) signal, usually recorded in human participants. EEG and MEG signals are less spatially specific than the LFP, but one might consider these external signals to represent some sort of spatial summation of many (local) LFPs.

Sensorimotor-related activity that scales to delay duration can also be observed in population measures such as the LFP, EEG and MEG. One example is the fronto-central contingent negative variation $(\mathrm{CNV})$. The $\mathrm{CNV}$ is a slow negative wave that develops between the cue and GO, mainly studied in the human EEG/MEG. Originally, Walter et al. [62] proposed that this eventrelated potential might reflect time estimation. Indeed, the CNV is sensitive to the duration of a delay or a stimulus presentation [63-66], and while the slope is steeper for shorter durations, the peak at the end of the duration remains unchanged in size during both explicit and implicit timing tasks, suggesting duration independence $[67,68]$. Overall, the timing-related dynamics of the CNV resembles the build-up spiking activity of single neurons described above. It was therefore proposed that both types of signals reflect the encoding of the timing of an upcoming event [67].

Oscillations are frequently observed in LFP, EEG and MEG signals. Power modulations in brain oscillations may be related to the degree of (rhythmic) spike synchronization [58] and/or the overall level of activity in neuronal populations [69]. Furthermore, the oscillation frequency may be related to the extent of neuronal networks [70-72] or the underlying excitation-inhibition balance [73-77]. Oscillations at different frequencies may therefore reflect different neuronal populations and/or network states. The typical oscillation frequency in motor cortex is within the so-called beta range ( 13-30 Hz; [78, 79]). Beta oscillations can synchronize over large networks, spanning multiple cortical [80-83] and sub-cortical areas [84, 85]. These oscillations are not strictly time-locked to signals or movements, as is the case for eventrelated potentials such as the $\mathrm{CNV}$ described above. However, beta oscillations are clearly 
modulated during sensorimotor behavior, being most prominent in epochs without overt movements (e.g., during delays) and during stable postures, and being minimal in power during movements as well as transiently following the presentation of sensory cues (see review in [86]). Motor cortical beta oscillations might reflect sensorimotor updating and planning processes [80, $81,83,86-89$ ], and a handful of observations in monkeys and humans suggest that they are also sensitive to timing processes $[39,47,90]$. In a recent study using rhythmic streams of auditory stimuli, Fujioka et al. [39] recorded MEG in human participants and found beta power to peak just before each sound event in several areas, including motor cortex, even though participants were only required to passively listen to the rhythmic streams. The authors suggested that this distributed pre-cue increase in beta power provides a mechanism for maintaining predictive timing. Arnal [91] proposed that this motor cortical sensory prediction might rely on the simulation of movements via an internal model, allowing the prediction of stimulus timing and its sensory consequences. However, it is also possible that this activity is a reflection of an automatic, covert movement preparation entrained by rhythmic stimuli. Such an automatic facilitation of gait by rhythmic stimuli has already been shown in Parkinsonian patients [92].

We observed similar effects in the motor cortical LFP of monkeys performing a visuomotor task with two possible delay durations ([47], see also [93]). Figure $2 d-g$ show the modulations in beta peak power and frequency between the cue and the GO signal, comparing short and long delay trials. In monkey $\mathrm{T}$ (Fig. 2d) the beta power increased substantially towards the GO signal, with a steeper increase in short than in long delay trials, following a post-cue transient power decrease. In this study [93] we demonstrated for the first time that not only peak power is task-modulated, but also the peak frequency of beta oscillations. Interestingly, even if beta power differed between short and long delay trials in only one of the two monkeys included in this study, beta frequency differed systematically between short and long delay trials for both monkeys (Fig. 2f, g). Following the cue, there was a transient increase in beta frequency, which was similar in onset and slope for short and long delay trials. Subsequent to this increase, the beta frequency slowly decreased towards the GO signal, with a steeper slope in short than long delay trials. Saleh et al. [90] recorded LFPs in primary motor cortex of a human patient who had to point to a spatial target with his chin. Five spatial cues were displayed successively, and he had to select either the second or the fourth cue in different trials. The power of beta oscillations peaked transiently before each spatial cue, with the highest pre-cue power for the correct cue. Furthermore, the beta power was phase-locked to slower delta oscillations $(0.5-1.5 \mathrm{~Hz})$ that matched the duration of the inter-cue intervals. In a similar way, Roux et al. [94] showed that the across-trial averaged LFPs systematically modulated as a slow wave during the delay period in relation to the temporal scheme of the task. This slow wave modulation also varied as a function of reaction time. In other words, the wave modulation varies in relation to the internal timing of delay duration of the animal from trial to trial; a similar effect was found for spiking activity of single neurons ([7]; see above).

Interestingly, modulations of durationselective beta oscillations appear to be similar to the $\mathrm{CNV}$ and the neuronal spiking activity. This suggests that many different types of gradual changes in motor cortical activity are duration-sensitive, with faster modulations for shorter durations, and a tendency to reach the same level of activation at the expected end of the estimated duration (e.g., the GO signal). Importantly, the scaling of activity modulations to duration was also found in human participants that were not receiving immediate reward on a single-trial basis. Thus, even though certain populations of neurons in motor areas are sensitive to reward magnitude and delay duration [19], there are also clear signs of similar timing sensitivity in these areas in the absence of an immediate reward. 
To conclude, motor cortical activity during movement preparation scales to delay duration and internal time estimation. This observation might reflect well-timed movement preparation, rather than an invariant timing mechanism. An alternative interpretation would be that motor cortex is part of a larger network coding time in a context-dependent manner.

\section{Precise Spike Synchrony and GO Signal Anticipation}

It is commonly accepted that sensorimotor functions are reflected in changes in firing rate in widely distributed populations of neurons [6]. However, the temporal coding hypothesis suggests that not only changes in firing rate but also precise spike timing constitute an important part of the representational substrate for perception and action. Precise spike timing here refers to spike synchronization or other precise spatiotemporal patterns of spike occurrences among neurons organized in functional groups, commonly called cell assemblies [95-98]. The concept of cell assemblies uses synchrony as an additional dimension to firing rate, as a candidate for information processing. The observation of precise spike synchrony between pairs of neurons [1] might be interpreted as activation of a functional cell assembly [99]. Motor cortical neurons synchronize their activity significantly at the moment of signal expectancy, indicating the end of an estimated delay duration, often without any corresponding firing rate modulation $[1,2$, 100]. Thus, behavioral timing modulates both spike synchrony and firing rate independently. Both experimental and theoretical studies point to the importance of synchronous spiking activity, particularly in a low firing-rate regime (e.g., [101]). Indeed, synchrony and firing rate might be complementary coding strategies, allowing for efficient computation with less activity through increased synchrony.

Assuming such a complementarity, the question arises whether improving the behavioral performance in a timing paradigm can alter the interplay between synchrony and firing rate. To study this, we quantified the strength of synchrony across pairs of neurons recorded in three monkeys performing a choice RT task, and compared it to the mean firing rate in the same neurons. In this task, the monkeys were asked to select the correct movement direction based on the delay duration between a cue and the GO signal, thus requiring correct estimation of elapsed time (see [3, 100]). Two targets of different colors were presented at the start of each trial (cue). A non-directional GO signal (auditory) was presented after a short or a long delay, randomly and with equal probability. The monkey learned to associate target color with delay duration.

We developed a method that provides the strength of synchronous spiking activity of an entire population of neuron pairs (see [100]) for the population quantification, for the statistics see the review by [102]. This method is based on the comparison between the numbers of empirical coincident spikes in pairs of neurons and the numbers of predicted coincident spikes, taking into account the instantaneous trial-bytrial firing rates of the neurons [102]. The difference between the number of observed versus predicted coincident spikes yields an analytical measure for each time-point and indicates the statistical significance of having more (or less) synchrony than expected by chance. This analysis can be done across all trials for all pairs of neurons, giving a time-resolved measure of population synchrony ([100]; see Fig. 3a).

In this study, the monkeys progressively improved their performance during the months of recording, significantly shortening RTs and/or reducing RT variability, suggesting an improved estimation of the delay durations [100]. We therefore split the population of recorded neurons into the first and last part of the recording period. Due to the task structure, the monkey expected (with $50 \%$ probability) a GO signal at the end of the short delay (ES; Fig. 3). In long delay trials, the synchrony strength of neuron-pairs recorded during the late sessions (black line) transiently increased after the expected GO signal (ES), exceeding the significance level of $p=0.01$ by far (dashed horizontal line). This brief increase 


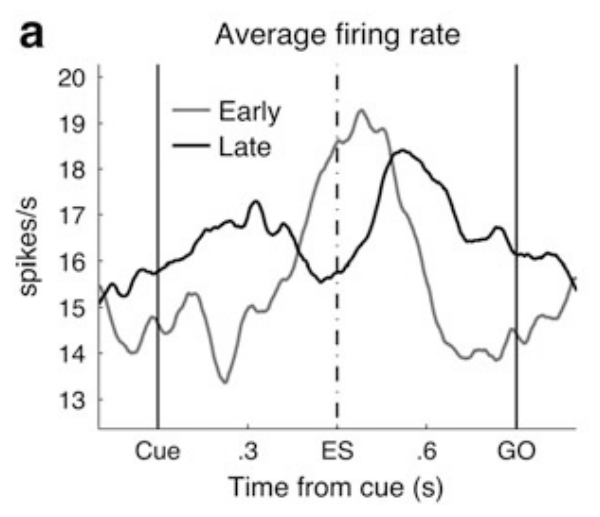

Fig. 3 Precise spike synchrony and GO signal expectancy. (a) Average firing rates (PETHs) of neurons recorded in one monkey during performance of a choice RT task, comparing early vs. late recording sessions (spanning several months). In this task, the monkey had to select the correct movement direction by estimating the delay duration between the cue and the GO signal (see main text). The GO signal could be presented either after a short or after a long delay duration. Here only long delay trials are shown, and ES (expected signal) represents the moment in which the animal expected the GO signal with $50 \%$ probability. (b) The data was analyzed with the "Unitary Event" technique [102, 149]. We developed a measure that provides the strength of synchronous spiking activity of an entire population of neuron pairs [100]. We used a sliding window of $100 \mathrm{~ms}$ duration (shifts of $5 \mathrm{~ms}$ ) moving through the entire length of the trial. We counted

of precise synchrony might provide an internal switch signal, triggered by correct time estimation, allowing a change in movement preparation (movement time and/or movement direction). In the same trial epoch, the mean population firing rate decreased in late compared to early sessions (Fig. 3b). We concluded that performance optimization in timing tasks might be achieved by increasing precise spike synchrony in relation to temporal expectancy, thereby boosting network efficiency. This may be accompanied by fewer spikes overall [100].

\section{Phasic Responses to the Cue}

We have so far considered motor cortical activity during the preparatory delay up to movement execution. However, motor cortex also exhibits short-latency signal-related phasic responses $(<200 \mathrm{~ms})$ to informative sensory cues ([103, 104]; examples in Fig. 4a, b). Pure execution-

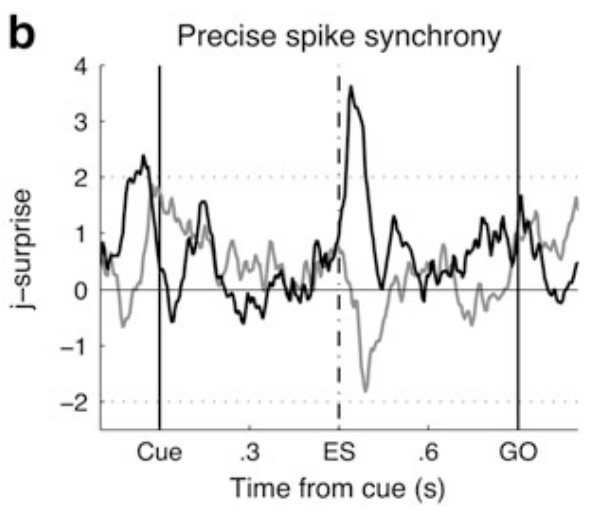

the number of empirical coincidences and calculated the number of coincidences that we would expect by chance by taking into account the instantaneous firing rate of the two neurons, for a temporal precision of up to $3 \mathrm{~ms}$. We then summed the result over all trials and pairs of neurons and calculated the statistical significance (joint-surprise) of the difference between empirical and predicted coincidences. Whenever the significance value exceeded the threshold (dashed line, $\mathrm{p}=0.01$ ), this defined an epoch in which significantly more coincidences occurred than would be expected by chance. Coincidences within such an epoch are called "unitary events" [149]. Values around zero indicate that there are as many synchronous spikes as expected by chance, positive (negative) values indicate more (less) coincidences. The data is shown for 42 vs. 45 neuron pairs, in early vs. late sessions, respectively (figure reproduced from [100])

related activity modulation patterns are more often observed in the central sulcus of M1, whereas delay- and signal-related activities are more common in PMd, the convexity of M1 being intermediate with respect to activity patterns [45, 103-108]. Auditory and visual cues are similarly efficient in eliciting signalrelated directional responses [109]. Roux et al. [3] showed that even the absence of an expected GO signal (i.e., an internal event) was followed by a phasic response in neurons that otherwise responded to cues. The cue does not need to be spatial in nature; a central symbolic cue also elicits a phasic response [110-114], though with a longer latency than for a simple peripheral cue $[107,113]$. However, a directionally noninformative cue does not elicit any phasic response in the motor cortex (e.g., [40, 103]).

Several lines of evidence suggest that the occurrence of a phasic response to a cue is related to movement preparation, rather than to a more general shift in spatial attention. First, neurons 

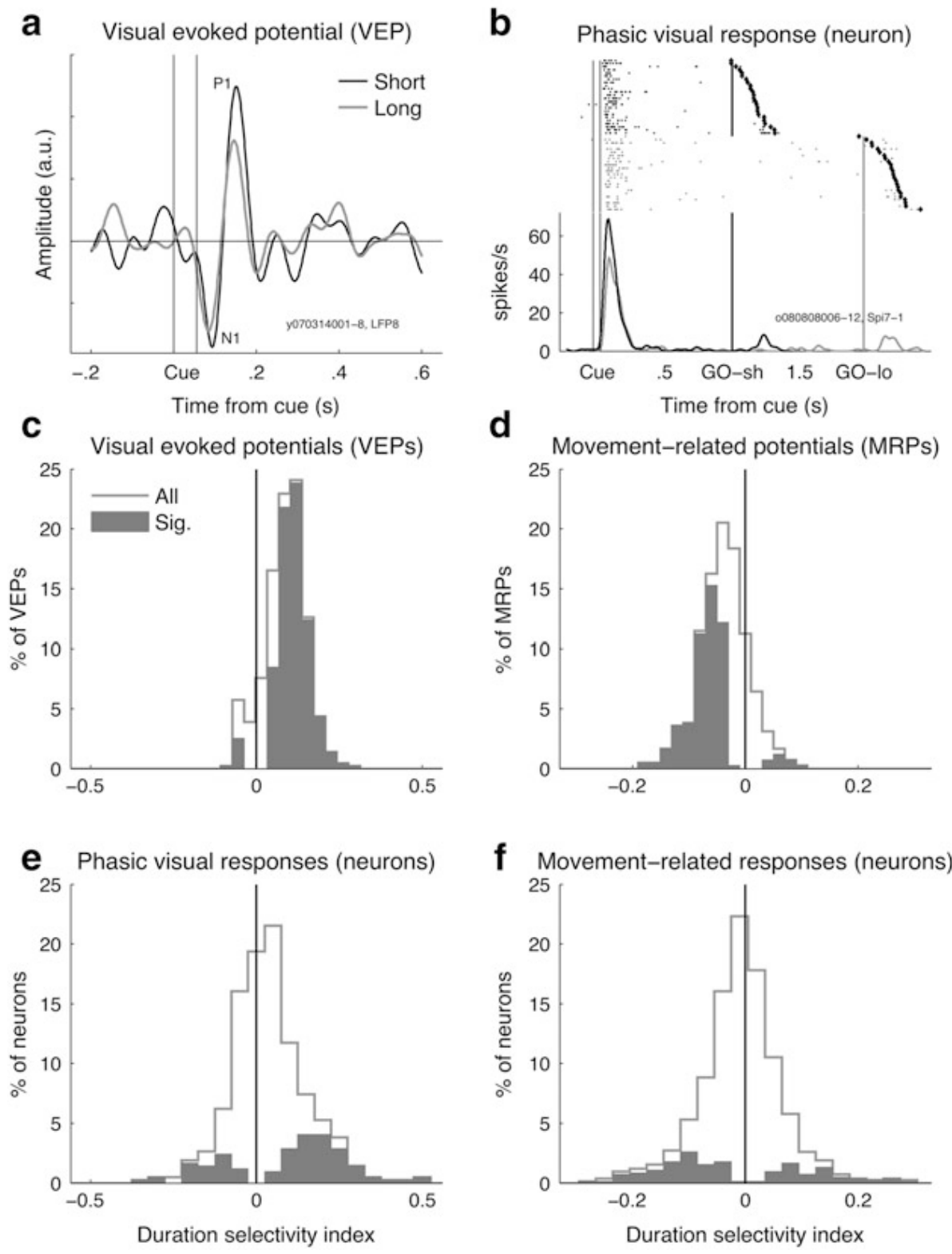

Fig. 4 Phasic responses to cues in motor cortex. (a) An example of a trial-averaged visual evoked potential (VEP) to the cue in one motor cortical LFP, comparing short and long delay trials. The labels N1 and P1 denote the two first typical components of the motor cortical VEP. (b) An example of a motor cortical neuron responding phasically to the cue, comparing short and long delay trials. The rasters are shown in the upper plot and the PETHs in the lower plot. The larger black dots to the right in the raster plot represent movement onset. (c, d) Distributions of duration selectivity indexes for all VEPs $(n=436)$ and movement-related potentials (MRPs; $n=419$ ), around movement onset, of two monkeys. The VEP and MRP sizes were calculated using the root-mean-square in single trial, including the $\mathrm{N} 1$ and $\mathrm{P} 1$ components of the VEP and the three most prominent components of the MRP. The duration selectivity index was defined as a contrast comparing average sizes in short and long delay trials [(short

- long)/(short + long)]. Positive values thus define short delay duration preference. As a reference value, an index of +0.33 would mean that the LFP amplitude is twice as big in short than in long trials. The outlines include all VEPs and MRPs and the filled bars only the VEPs and MRPs with a significant difference in size between short and long delay trials $(n=330$ and 217 for VEPs and MRPs, respectively). Significance in individual VEPs and MRPs was tested with a two-way ANOVA (delay duration and movement direction as factors, $\mathrm{p}<0.05$ ). The medians of the distributions of significant VEPs and MRPs are +0.11 and -0.06 , respectively, significantly shifted away from zero (Wilcoxon signed rank test; $\mathrm{p}<<0.001$ for VEPs and MRPs). See Kilavik et al. [108] for more details on recording and analysis techniques (data from [108]). (e, f) Distributions of duration selectivity indexes for all neurons in two monkeys with a phasic response to the cue $(n=418)$ and for all 
with a phasic response to the GO signal may show as well a phasic response to the cue [106]. Second, this phasic response to the GO signal may decrease strongly or even disappear if the preceding cue provided already spatial information ("pre-processing neuron" of 6,45, 115). Third, neurons in PMd are much more responsive (and selective) to cues indicating the target of a movement than to cues indicating the target of a shift in attentional focus [40, 116-119]. Last, the signalrelated activity in PMd reflects the planned action rather than the characteristics of the cue itself (shape, color, location) [111, 114, 120].

The earliest modulations in motor cortical activity start around 50-60 ms after cue onset $[103,104,108]$ and are generally less spatially selective than the later ones [104]. Signal-related activity with slightly shorter latencies might reflect a first wave of information concerning targets of potential movements. This preliminary information becomes more refined as more complex processing of the cue continues to unfold. For instance, in both M1 and PMd the vast majority of initial phasic responses are selective to the direction of the target and not to the direction of the movement $[12,121,122]$. Even partial information about movement direction elicits a phasic response, though with a smaller amplitude than after complete directional information [107, 123]. Finally, the very early signal-related activity is relatively unaffected by the condition of a GO-NOGO task, unlike the subsequent delayactivity [124-126].

The signal-related phasic response is as sensitive to delay duration as the subsequent delay activity during movement preparation [3, 108]. Figure $4 \mathrm{a}, \mathrm{b}$ show examples of a LFP visual evoked potential (VEP) (A) and a neuron with a phasic change in activity (B), both recorded in motor cortex as a response to the cue $[41,108]$. In both cases, the amplitudes of the phasic response were larger in short delay trials than in long delay trials. This was true for a majority of motor cortical LFP VEPs (77 \%, see Fig. 4c; [108]). Interestingly, this result is complementary to the fact that movement-related potentials (MRPs) observed during movement execution are larger in long delay than in short delay trials (significant in $52 \%$; Fig. 4d; [108]). We found that the phasic cue response in $25 \%$ of these neurons was selective to delay duration (Fig. 4e; [41]), preferring, as in VEPs, mainly short delay trials. The influence of delay duration on the spiking activity during movement execution was less clear (17\% significant; Fig. 4f), even though a majority had higher firing rates in long delay trials, similarly to MRPs.

If the early phasic activity after the cue in motor cortex reflects a pre-processing mechanism, we can conclude that pre-processing is more prominent in short delay trials, observable in overall higher firing rates and larger VEPs. This is complementary to a lower activity in short delay trials during movement execution, particularly noticeable in the MRPs. Beyond this, we can currently only speculate on why there is such a large quantitative difference between the spiking activities and MRPs around movement onset. It is important to note that the LFP not only reflects sub-threshold synaptic activity, but also population activity. This means that a weak, but consistent, effect of delay duration in a sufficiently large population of neurons may be observed more clearly in the LFP. Importantly, the modulation of evoked LFP responses by delay duration suggests that there is a high degree of flexibility in the movement preparation process, which is significantly
Fig. 4 (Continued) neurons, independently of their activity pattern around movement onset $(n=847)$. The duration selectivity index was calculated as described above, using mean spike counts in $200 \mathrm{~ms}$ large windows, after cue onset and around movement onset. Significance was tested with a two-way ANOVA (delay duration and movement direction as factors; $\mathrm{p}<0.05)$. Medians for the sub-sets of significant neurons (filled gray bars) are +0.14 for the phasic visual responses $(n=105)$ and -0.06 for the movement-related responses $(n=143)$, in both cases significantly shifted away from zero (Wilcoxon signed rank test; $\mathrm{p}<<0.001$ and $\mathrm{p}=0.017$, respectively) (unpublished data from $\mathrm{J}$ Confais, BE Kilavik, A Ponce-Alvarez and A. Riehle) 
influenced by the knowledge of the available time prior to movement execution [108].

Earlier we described how neuronal activity during movement preparation is scaled to delay duration and internal time estimation. Here we show that this scaling can also be extended to signal processing and movement execution epochs. One appealing interpretation is that for shorter delays it might be optimal to complete movement preparation early directly after cue presentation, whereas for longer delays it may become more 'economic' to encode only general aspects of the movement early in the delay (e.g., movement direction or goal) and to finalize more detailed aspects of the movement prior to and during its execution.

\section{Part 2: Timing During Cue Anticipation}

In the first part of this chapter, we described how the timing of a motor task profoundly shapes the activity of motor cortex during movement preparation. In this part, we will see how available information about time is also used to predict the timing of an upcoming cue, which carries relevant information for movement preparation. In this context, an anticipatory pattern of activity preceding the cue onset can be observed in motor cortical areas, even in absence of motor preparation.

In the movement preparation paradigm (see Fig. 1) a delay is used to temporally separate the moment when the subject is provided with information about the desired action (for example, a spatial cue indicating the position that should be pointed towards) and the moment when this action has to be performed (GO signal). However, in most experimental protocols, the subject can also estimate the duration preceding the informative cue. The timing of the cue can be predicted by keeping the preceding delay fixed in all trials (e.g., [9]) or by adding a "pre-cue" to indicate the length of the upcoming delay in each trial (e.g., [41]). Since the movement preparation paradigm was first implemented, it was regularly reported that a fraction of motor cortical neurons modulate their firing rate well before the presentation of the cue. However, this was only described as a side note $[40,106,107,109,111$, $122,127]$ or examples of neurons with such an activity were shown [3, 103], but only a few studies examined this type of anticipatory activity directly $[9,10,41]$. Interestingly, this type of activity has been described in a wide range of brain structures, such as the caudate nucleus [128-130], the prefrontal cortex [8, 131], and the somatosensory cortex [37]. In this part we will review the main characteristics of this "cueanticipatory activity" by focusing on motor preparation in motor cortical areas, and will suggest a possible functional significance. For convenience purposes, we refer to the neurons showing this type of activity as "cue-anticipatory neurons" in this chapter.

\section{Prevalence of Cue-Anticipatory Activity in Frontal Areas}

All works describing an anticipatory activity used a fixed or highly predictable pre-cue delay. Vaadia et al. [10] trained monkeys extensively in a task that included a fixed 3 s delay preceding the cue, whereas in our task the pre-cue delay duration was indicated in advance to the animal by an auditory signal [41]. We observed different patterns of motor cortical spiking activity during this delay: some neurons increase their activity preceding the cue (see Fig. 5b), others decrease it (Fig. 5a), and the remaining neurons do not modulate. Even if the ratios vary from study to study, most studies report more neurons with increasing than decreasing firing rates $[10,41,106]$. However, the ratio of neurons with anticipatory activity seems to depend on the cortical location. Most studies in frontal areas showed the strongest representation of anticipatory activity in PMd. Crammond and Kalaska [106] showed a higher percentage of anticipatory neurons in PMd than in M1. Additionally, they demonstrated that the ratio of anticipatory neurons with increasing versus decreasing activity changed with the distance to the central sulcus. The majority of anticipatory neurons increased their activity in PMd and in 
a Pre-cue anticipatory decreasing neuron
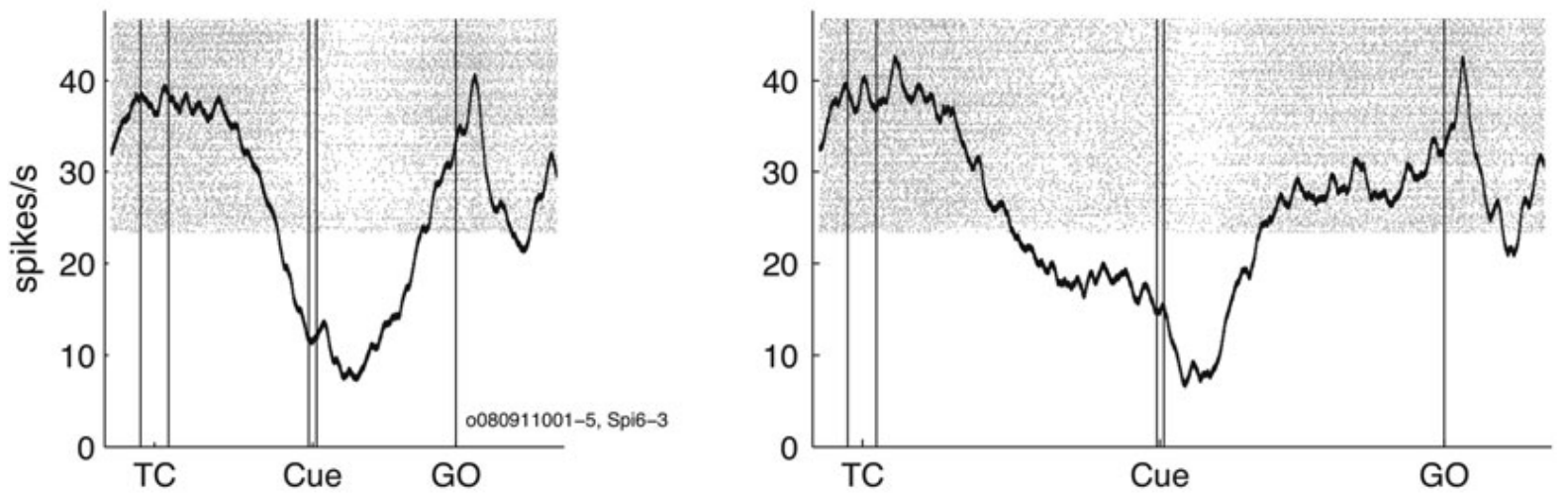

b

Pre-cue anticipatory increasing neuron
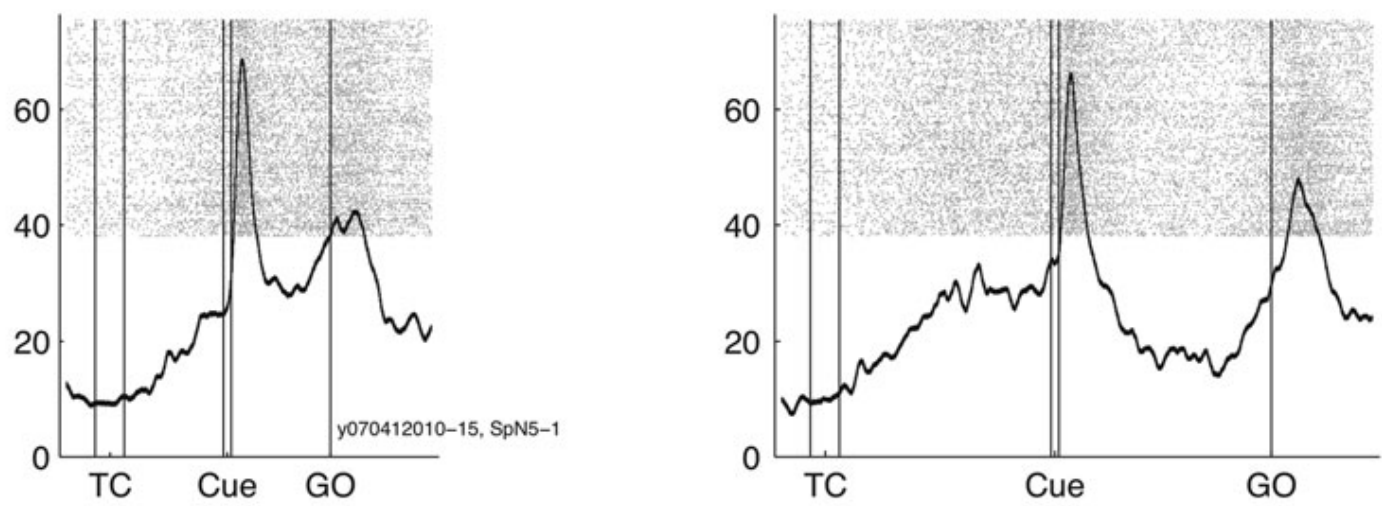

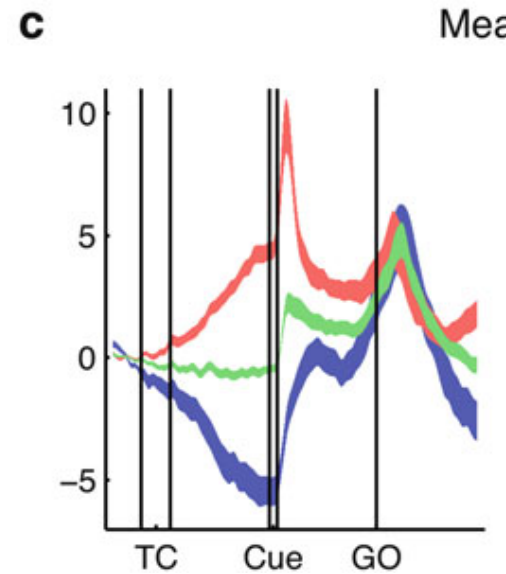

Fig. 5 Pre-cue anticipatory activity in motor cortex. (a, b) Raster plots and PETHs of two example neurons with pre-cue anticipatory activity (short delay trials on the left, long delay trials on the right). The trials are arranged chronologically. In a, please note the suppression of activity following the cue for this neuron with pre-cue decreasing activity. In $\mathbf{b}$, note the phasic response to cue in this neuron with pre-cue increasing activity. (c) Averaged activity of all neurons in one monkey $( \pm$ sem),

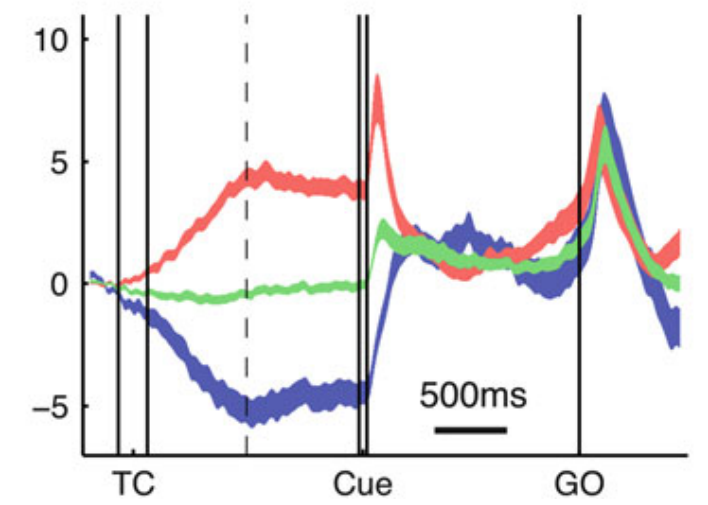

classified according to pattern of pre-cue anticipatory activity. The baseline activity (final $200 \mathrm{~ms}$ before TC) of each neuron is subtracted prior to averaging. Pre-cue increasing neurons in red $(\mathrm{n}=129)$, pre-cue decreasing in blue $(\mathrm{n}=95)$, and non-anticipatory neurons in green $(\mathrm{n}=228)$. TC: time cue presented for $200 \mathrm{~ms}$, indicating delay durations, Cue: spatial cue presented for $55 \mathrm{~ms}$, indicating target location, GO: directionally noninformative GO signal (data from [41]) 
the rostral part of M1, whereas all anticipatory neurons recorded in the caudal (sulcal) part of M1 decreased their activity. Interestingly, this caudal zone of M1 has the highest density of direct cortico-motoneuronal projections [132]. Hoshi and Tanji [111] showed more anticipatory neurons in PMd than in the ventral part of the premotor cortex (PMv). They also showed that anticipatory neurons in PMd were more sensitive to the expected information contained in the cue than those in PMv. Di Pellegrino et al. [40] and Vaadia et al. [10] showed similar differences between PMd and prefrontal cortex (PF), whereby anticipatory activity was observed more often in PMd than in PF neurons, accompanied by an increased sensitivity to the expected cue information in PMd neurons. In contrast, we did not find any difference in the proportions of anticipatory neurons between PMd and M1, but this may be due to the fact that our recording chamber only captured the rostral part of M1 [41].

\section{Influence of Expected Cue Information}

As mentioned above, some studies found an effect of different cue aspects on the anticipatory activity. In particular, three studies showed an influence of the expected information carried by the cue, albeit to a different extent. Vaadia et al. [10] used a block-wise presented task in which the cue in one block either indicated the target of the movement or was non-informative. They showed that a small fraction of the neurons changed selectively their activity preceding the cue, depending on the block. Similarly, in Di Pellegrino and Wise [40], the cue indicated either the movement target or a shift in attentional focus, irrespective of the movement target. Two-thirds of the anticipatory neurons modulated their firing rate depending on the information carried by the cue, with generally more activity in the "movement target" blocks than in the "attention only" blocks. Finally, Hoshi and Tanji [111] showed that the activity of more than $10 \%$ of the anticipatory PMd neurons significantly changed their activity depending on whether the cue was expected to contain movement target information or to contain information about which arm to use. Interestingly, the expected reward can also modulate the cue anticipatory activity. Vaadia et al. [10] added a condition in which the trials were rewarded at random and showed that a subsample of anticipatory neurons stopped responding after several unrewarded trials in a row.

Although most of the anticipatory neurons in these studies show an increasing activity, some display a decreasing pattern. We will now describe how these patterns of anticipatory activity are predictive for the firing rate modulations during movement preparation $[9,41]$.

\section{Relationship Between Cue Anticipation and Movement Preparation Activity}

We recorded motor cortical single neuron activity in a delayed center-out reaching task [41]. Each trial contained two successive delays of equal duration, indicated at the beginning of each trial by an auditory cue. At the end of the first delay, a spatial cue indicating the direction of the upcoming movement was briefly flashed. At the end of the second delay, the (noninformative) GO signal requested to reach to the cued target. This task was conceptually different during the two delays: during the first delay, the monkeys used the temporal information provided by the temporal cue to accomplish at a given time a visual detection task, whereas they had to time and prepare the upcoming movement during the second delay. In about $40 \%$ of the neurons the activity was modulated during the first delay and these neurons were therefore classified as anticipatory. From this group, $60 \%$ increased and $40 \%$ decreased their activity (examples in Fig. 5). When comparing the averaged activity of these different neuronal populations, a striking difference could be seen during the second delay (Fig. 5c). Following the spatial cue, the pre-cue anticipatory neurons with increasing activity showed an early phasic response, whereas the neurons with decreasing activity were largely suppressed during the same epoch. This confirms the finding by Mauritz and 


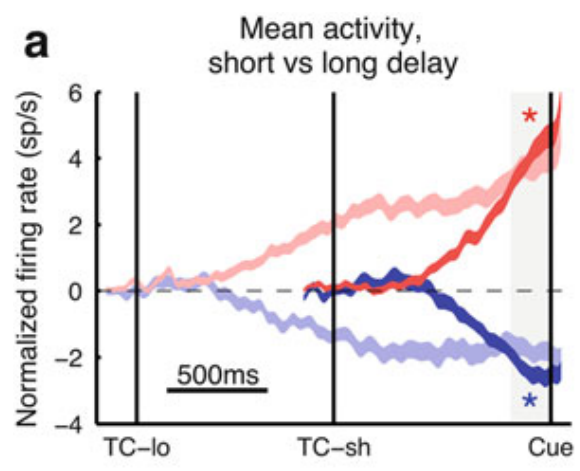

Fig. 6 Influence of delay duration on pre-cue anticipatory activity. (a) Mean activity $( \pm$ sem) of the cueanticipatory increasing (red, $\mathrm{n}=170$ ) and decreasing neurons (blue, $\mathrm{n}=113$ ) recorded in one monkey (monkey M). The activity in short and long delay trials are in dark and light colors, respectively. TC-lo and TC-sh is the onset of the time cue (200 ms duration) in long and short delay trials, informing about the delay duration. The data is aligned to Cue onset. The baseline activity has been subtracted. The light gray rectangle indicates the epoch used to compute the significance of the difference between short- and long-delay trials. (b) Distributions of

Wise [9] that almost all anticipatory neurons with an increasing activity show a phasic, shortlatency response to the spatial cue. In addition, we show that the activity of the decreasing anticipatory neurons is suppressed after the cue. Simply put, the pre-cue increasing neurons are more active during the post-cue cue epoch than during the movement, whereas the pre-cue decreasing neurons are more active during movement execution [41].

\section{Delay Duration Effects During Cue Anticipation}

If the cue occurs probabilistically at one out of several, discrete points in time, the subject might expect a cue after shorter durations, even when the cue does not appear. This expectation has been shown in neurons that increase their activity until the time of the expected cue and then suddenly decrease their activity when the cue does not occur $[3,7,9,133]$. In other words, if a neuron is classified as "increasing" during a short delay, it may change its pattern during a long delay [10].

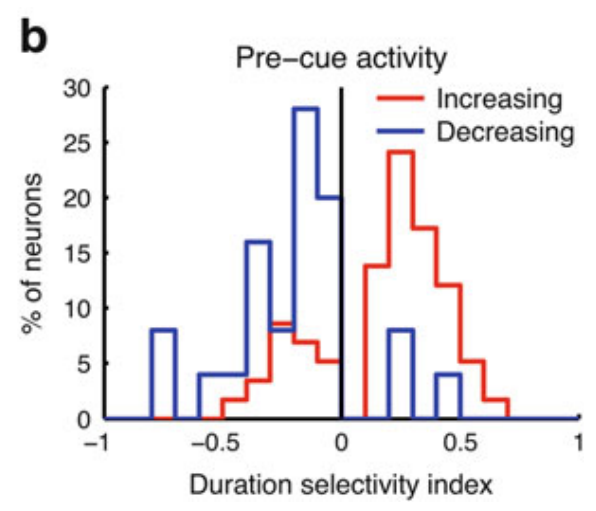

the indexes of duration selectivity comparing short and long delay trials, using the pre-cue epoch marked in a. The index is defined as [(short - long)/(short + long)]. Positive values indicate a higher activity in short delay trials. Only the neurons with a significant difference (Mann-Whitney U test, $\mathrm{p}<0.05)$ are shown $(\mathrm{n}=58$ and 25 for increasing and decreasing neurons, in red and blue respectively). The medians of both distributions are significantly shifted away from 0 (Wilcoxon signed rank test, $\mathrm{p}<0.05$ ) (unpublished data from J Confais, BE Kilavik, A Ponce-Alvarez and A. Riehle)

Alternatively, the duration of the delay may be known with certainty, as in the study of Confais et al. [41]. Here, it becomes evident that the modulation depth in both increasing and decreasing anticipatory neurons is larger in short than in long delay trials (Fig. 6a). In the first part of this chapter, we described how the neuronal response to the spatial cue depended on delay duration, with both the phasic spiking activity and the VEP of the LFPs being larger in short than in long delay trials (Fig. 4). Most anticipatory neurons with an increasing pre-cue activity also show a phasic response to the cue. Therefore, the larger modulation of pre-cue firing rate observed in these neurons in short delay trials might mediate the subsequent larger responses to the cue. Furthermore, the differences in firing rate in short and long delay trials are clearly opposite for the increasing and decreasing sub-populations (Fig. 6b). This "mirrored" modulation of the precue firing rate suggests that the two subpopulations of neurons have complementary roles. 

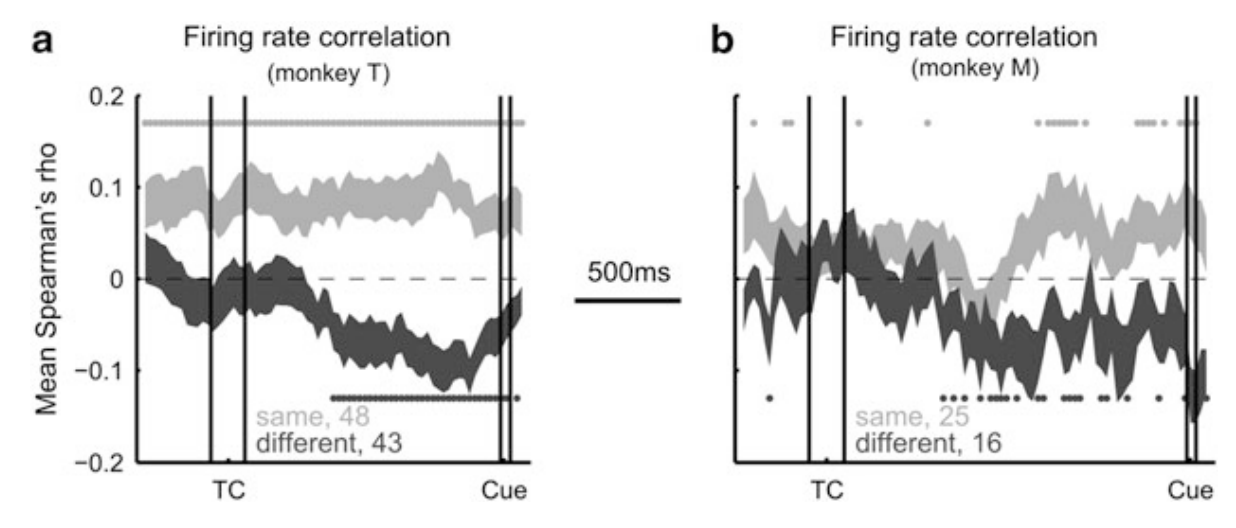

Fig. 7 Trial-by-trial correlations between cueanticipatory neurons. The curves represent the mean coefficient of correlation $( \pm \mathrm{sem})$, for pairs of cue-anticipatory neurons with the same activity pattern (both increasing or decreasing, in light grey) and with opposite activity patterns (one increasing and one decreasing, in dark grey). We used a sliding window of $250 \mathrm{~ms}$ to guarantee a sufficient amount of spikes, and selected only neurons recorded on different electrodes. In each window, we performed a Spearman rank correlation between the spike counts across trials of each neuron pair. The correlation coefficients were transformed in Fisher $\mathrm{z}$ before

\section{Possible Functional Role(s) of Pre-cue Anticipatory Activity}

The anticipatory activity could be the reflection of attentional processes, as already shown in PMd [134, 135]. However, we did not find any difference in the pre-cue activity between correct and error trials, i.e., selecting the wrong cue, presumably because of attentional fluctuations [41]. Alternatively, it could reflect a general timing process. Durstewitz [49] proposes that climbing activity, like the one observed before the spatial cue, could be a possible substrate of time estimation. Furthermore, several fMRI studies show activation of the premotor cortex during tasks involving time estimation [136, 137]. However, as we discussed in Part 1, a timing mechanism based on climbing activity would need a fixed onset and a slope that differs according to delay duration. Yet, Fig. 5c (right panel) shows that the activity during the second part of the first delay in long trials is flat until the cue appears. This suggests that even if such an activity depends on the ability of the animal to estimate the delay duration, it is unlikely that it reflects timing per se. Another hypothesis would be that averaging across pairs, and then transformed back. We only analyzed the pre-cue delay in long delay trials. The diamonds at the top and bottom of the plots indicate time bins in which the mean coefficient of correlation is significantly different from 0 (Wilcoxon signed-rand test, $\mathrm{p}<0.05$ ). Only long-delay trials are shown. (a) Monkey $\mathrm{T}, \mathrm{n}=48$ and 43 pairs of neurons of the same and different category, respectively. (b) Monkey $\mathrm{M}, \mathrm{n}=25$ and 16 pairs of neurons of the same and different category, respectively (unpublished data from J Confais, BE Kilavik, A Ponce-Alvarez and A Riehle)

the anticipatory activity uncovers two parallel processes complementary to each other. One would facilitate the response to the spatial cue through an additive gain (as it is the case in the caudate nucleus, e.g., [129]), whereas the other would suppress a premature motor response, since the movement execution has to be withheld until the GO signal appears ("proactive volitional inhibition", e.g., [138], "impulse control", e.g. [139], "proactive control", see [140] for a review). Such an interpretation is supported by a modeling study of Moody and Wise [141] showing that an anticipatory activity emerges in some neurons before the cue during a match-tosample task, but only if the cue timing is predictable. Removing these neurons either leads to false negative or false positive responses. The authors interpret this result as evidence for two parallel processes before cue occurrence, while preventing a premature response.

One prediction can be drawn from the idea that two sub-populations compensate each other's activity. The neuronal activities within a sub-population (e.g., neurons increasing their activity) would tend to positively co-vary trialby-trial, whereas the neuronal activities of the 
two different subpopulations would negatively co-vary. To test this hypothesis, we computed the mean trial-by-trial correlation ("noise correlation", see [142]) between spiking activities of pairs of neurons with the same anticipatory activity pattern and with opposite anticipatory activity, recorded simultaneously (Fig. 7). The mean trial-by-trial correlation of firing rates is dramatically different for pairs within the same category than for pairs from different categories. Neurons with the same pattern of pre-cue anticipatory activity are mainly positively correlated. Neurons with different patterns are not correlated initially, but become increasingly negatively correlated during the pre-cue delay. This again supports the idea that the two sub-populations play complementary roles in order to facilitate cue detection, while preventing premature motor output. These two sub-populations do not only negatively co-vary in relation to the pre-cue delay duration in their averaged activity (Fig. 6), but are also dynamically co-adjusted on a trial-by-trial basis (Fig. 7).

To conclude, the cue-anticipatory activity may reflect prospective facilitation of cue processing concurrent with proactive movement inhibition. As mentioned initially, cueanticipatory activity is more prevalent in PMd than in other frontal areas. This idea is supported by studies linking PMd to the processing of spatial cues (e.g., [118]) and movement inhibition [143-145]. With this in mind, our results could therefore be interpreted as PMd playing a key role in the pre-setting of these processes.

\section{Summary and Conclusion}

The accurate estimation of time intervals is an essential aspect of motor performance; it is at the core of any anticipatory behavior. We have shown that timing processes are indeed represented in motor cortical single neuron and population activity, in a manner that is strongly dependent on context. It is tempting to speculate that the increase of firing rate and spike synchrony at specific task moments reflect a cognitive state; an internal representation of the precise timing of an expected event. This could favor the idea that timing, to some extent, is a constituent of currently active networks, and is therefore a distributed brain process. However, it is not clear if time itself is represented in the brain as an invariant process, separable from other processes, such as cue anticipation or movement preparation. The characteristics of the single neuron climbing activity observed during movement preparation in motor cortical areas suggest its origin upstream from the recorded neuron. Additionally, the effects of implicit and explicit timing in the activity of single neurons in motor cortex are very similar. It is difficult to discern in motor cortical activity whether different mechanisms are involved when timing is only implicitly used to improve performance or when timing is a crucial component of the task.

To conclude, if time estimation is a process independent of contextual features such as probability or movement preparation, then the signatures of time that we have described here are more likely the result of time estimation and not the time estimation process itself (see also the discussion in [3]). The question still remains open whether a general, contextindependent neuronal correlate of time estimation exists (e.g., [146-148]).

Acknowledgement We thank Marcel de Haan for critically reading the manuscript. This work was supported by a grant from Fondation pour la Recherche Médicale (FRM) to J.C.

\section{References}

1. Riehle A, Grün S, Diesmann M, Aertsen A. Spike synchronization and rate modulation differentially involved in motor cortical function. Science. 1997;278(5345):1950-3.

2. Riehle A, Grammont F, Diesmann M, Grün S. Dynamical changes and temporal precision of synchronized spiking activity in monkey motor cortex during movement preparation. J Physiol Paris. 2000;94(5-6):569-82.

3. Roux S, Coulmance M, Riehle A. Context-related representation of timing processes in monkey motor cortex. Eur J Neurosci. 2003;18(4):1011-6. 
4. Janssen P, Shadlen MN. A representation of the hazard rate of elapsed time in macaque area LIP. Nat Neurosci. 2005;8(2):234-41.

5. Requin J, Brener J, Ring C. Preparation for action. In: Jennings RR, Coles MGH, editors. Handbook of cognitive psychophysiology: central and autonomous nervous system approaches. New York: Wiley; 1991.

6. Riehle A. Preparation for action: one of the key functions of the motor cortex. In: Riehle A, Vaadia E, editors. Motor cortex in voluntary movements: a distributed system for distributed functions. Boca Raton: CRC; 2005.

7. Renoult L, Roux S, Riehle A. Time is a rubberband: neuronal activity in monkey motor cortex in relation to time estimation. Eur J Neurosci. 2006;23 (11):3098-108.

8. Niki H, Watanabe M. Prefrontal and cingulate unit activity during timing behavior in the monkey. Brain Res. 1979;171(2):213-24.

9. Mauritz KH, Wise SP. Premotor cortex of the rhesus monkey: neuronal activity in anticipation of predictable environmental events. Exp Brain Res. 1986;61 (2):229-44.

10. Vaadia E, Kurata K, Wise SP. Neuronal activity preceding directional and nondirectional cues in the premotor cortex of rhesus monkeys. Somatosens Mot Res. 1988;6(2):207-30.

11. Lucchetti C, Bon L. Time-modulated neuronal activity in the premotor cortex of macaque monkeys. Exp Brain Res. 2001;141(2):254-60.

12. Ghose GM, Maunsell JHR. Attentional modulation in visual cortex depends on task timing. Nature. 2002;419(6907):616-20.

13. Leon MI, Shadlen MN. Representation of time by neurons in the posterior parietal cortex of the macaque. Neuron. 2003;38(2):317-27.

14. Brody CD, Hernández A, Zainos A, Romo R. Timing and neural encoding of somatosensory parametric working memory in macaque prefrontal cortex. Cereb Cortex. 2003;13(11):1196-207.

15. Akkal D, Escola L, Bioulac B, Burbaud P. Time predictability modulates pre-supplementary motor area neuronal activity. Neuroreport. 2004;15(8):1283-6.

16. Lucchetti C, Ulrici A, Bon L. Dorsal premotor areas of nonhuman primate: functional flexibility in time domain. Eur J Appl Physiol. 2005;95(2-3):121-30.

17. Tsujimoto $S$, Sawaguchi $T$. Neuronal activity representing temporal prediction of reward in the primate prefrontal cortex. J Neurophysiol. 2005;93 (6):3687-92.

18. Roesch MR, Olson CR. Neuronal activity in primate orbitofrontal cortex reflects the value of time. J Neurophysiol. 2005;94(4):2457-71.

19. Roesch MR, Olson CR. Neuronal activity dependent on anticipated and elapsed delay in macaque prefrontal cortex, frontal and supplementary eye fields, and premotor cortex. J Neurophysiol. 2005;94 (2):1469-97.
20. Genovesio A, Tsujimoto S, Wise SP. Neuronal activity related to elapsed time in prefrontal cortex. J Neurophysiol. 2006;95(5):3281-5.

21. Maimon G, Assad JA. A cognitive signal for the proactive timing of action in macaque LIP. Nat Neurosci. 2006;9(7):948-55.

22. Maimon G, Assad JA. Parietal area 5 and the initiation of self-timed movements versus simple reactions. J Neurosci. 2006;26(9):2487-98.

23. Shuler MG, Bear MF. Reward timing in the primary visual cortex. Science. 2006;311(5767):1606-9.

24. Kalenscher T, Ohmann T, Windmann S, Freund N, Güntürkün $\mathrm{O}$. Single forebrain neurons represent interval timing and reward amount during response scheduling. Eur J Neurosci. 2006;24(10):2923-31.

25. Lebedev MA, O'Doherty JE, Nicolelis MAL. Decoding of temporal intervals from cortical ensemble activity. J Neurophysiol. 2008;99(1):166-86.

26. Schneider BA, Ghose GM. Temporal production signals in parietal cortex. PLoS Biol. 2012;10(10): e1001413.

27. Coull J, Nobre A. Dissociating explicit timing from temporal expectation with fMRI. Curr Opin Neurobiol. 2008;18(2):137-44.

28. Lee IH, Assad JA. Putaminal activity for simple reactions or self-timed movements. J Neurophysiol. 2003;89(5):2528-37.

29. Mita A, Mushiake H, Shima K, Matsuzaka Y, Tanji J. Interval time coding by neurons in the presupplementary and supplementary motor areas. Nat Neurosci. 2009; 12(4):502-7.

30. Shinomoto S, Omi T, Mita A, Mushiake H, Shima K, Matsuzaka Y, et al. Deciphering elapsed time and predicting action timing from neuronal population signals. Front Comput Neurosci. 2011;5:29.

31. Zarco W, Merchant H, Prado L, Mendez JC. Subsecond timing in primates: comparison of interval production between human subjects and rhesus monkeys. J Neurophysiol. 2009;102(6):3191-202.

32. Merchant H, Zarco W, Pérez O, Prado L, Bartolo R. Measuring time with different neural chronometers during a synchronization-continuation task. Proc Natl Acad Sci U S A. 2011;108(49):19784-9.

33. Merchant H, Pérez O, Zarco W, Gámez J. Interval tuning in the primate medial premotor cortex as a general timing mechanism. J Neurosci. 2013;33 (21):9082-96.

34. Merchant H, Georgopoulos AP. Neurophysiology of perceptual and motor aspects of interception. J Neurophysiol. 2006;95(1):1-13.

35. Sakurai Y, Takahashi S, Inoue M. Stimulus duration in working memory is represented by neuronal activity in the monkey prefrontal cortex. Eur J Neurosci. 2004;20(4):1069-80.

36. Genovesio A, Tsujimoto S, Wise SP. Feature- and order-based timing representations in the frontal cortex. Neuron. 2009;63(2):254-66.

37. Meftah E-M, Bourgeon S, Chapman CE. Instructed delay discharge in primary and secondary 
somatosensory cortex within the context of a selective attention task. J Neurophysiol. 2009;101 (5):2649-67.

38. Van Ede F, de Lange F, Jensen O, Maris E. Orienting attention to an upcoming tactile event involves a spatially and temporally specific modulation of sensorimotor alpha- and beta-band oscillations. J Neurosci. 2011;31(6):2016-24.

39. Fujioka T, Trainor LJ, Large EW, Ross B. Internalized timing of isochronous sounds is represented in neuromagnetic $\beta$ oscillations. J Neurosci. 2012;32(5):1791-802.

40. Di Pellegrino G, Wise SP. Visuospatial versus visuomotor activity in the premotor and prefrontal cortex of a primate. J Neurosci. 1993;13(3): 1227-43.

41. Confais J, Kilavik BE, Ponce-Alvarez A, Riehle A. On the anticipatory precue activity in motor cortex. $\mathrm{J}$ Neurosci. 2012;32(44):15359-68.

42. Weinrich M, Wise SP. The premotor cortex of the monkey. J Neurosci. 1982;2(9):1329-45.

43. Romo R, Schultz W. Neuronal activity preceding self-initiated or externally timed arm movements in area 6 of monkey cortex. Exp Brain Res. 1987;67 (3):656-62.

44. Schultz W, Romo R. Neuronal activity in the monkey striatum during the initiation of movements. Exp Brain Res. 1988;71(2):431-6.

45. Crammond DJ, Kalaska JF. Prior information in motor and premotor cortex: activity during the delay period and effect on pre-movement activity. J Neurophysiol. 2000;84(2):986-1005.

46. Lebedev MA, Wise SP. Oscillations in the premotor cortex: single-unit activity from awake, behaving monkeys. Exp Brain Res. 2000;130(2):195-215.

47. Kilavik BE, Riehle A. Timing structures neuronal activity during preparation for action. In: Nobre AC, Coull JT, editors. Attention and time. Oxford: Oxford University Press; 2010. p. 257-71.

48. Durstewitz D. Self-organizing neural integrator predicts interval times through climbing activity. J Neurosci. 2003;23(12):5342-53.

49. Durstewitz D. Neural representation of interval time. Neuroreport. 2004;15(5):745-9.

50. Reutimann J, Yakovlev V, Fusi S, Senn W. Climbing neuronal activity as an event-based cortical representation of time. J Neurosci. 2004;24(13):3295-303.

51. Okamoto H, Isomura Y, Takada M, Fukai T. Temporal integration by stochastic recurrent network dynamics with bimodal neurons. J Neurophysiol. 2007;97(6):3859-67.

52. Gibbon J. Scalar expectancy theory and Weber's law in animal timing. Psychol Rev. 1977;84(3):279-325.

53. Lowenstein G, Elster J. Choice over time. New York: Russell Sage; 1992. 399 p.

54. Berdyyeva TK, Olson CR. Relation of ordinal position signals to the expectation of reward and passage of time in four areas of the macaque frontal cortex. $\mathrm{J}$ Neurophysiol. 2011;105(5):2547-59.
55. Mitzdorf U. Current source-density method and application in cat cerebral cortex: investigation of evoked potentials and EEG phenomena. Physiol Rev. 1985;65(1):37-100.

56. Mitzdorf U. Properties of cortical generators of event-related potentials. Pharmacopsychiatry. 1994;27(2):49-51.

57. Logothetis NK, Kayser C, Oeltermann A. In vivo measurement of cortical impedance spectrum in monkeys: implications for signal propagation. Neuron. 2007;55(5):809-23.

58. Denker M, Roux S, Lindén H, Diesmann M, Riehle A, Grün S. The local field potential reflects surplus spike synchrony. Cereb Cortex. 2011;21(12):2681-95.

59. Lindén H, Tetzlaff T, Potjans TC, Pettersen KH, Grün S, Diesmann M, et al. Modeling the spatial reach of the LFP. Neuron. 2011;72(5):859-72.

60. Rasch MJ, Gretton A, Murayama Y, Maass W, Logothetis NK. Inferring spike trains from local field potentials. J Neurophysiol. 2008;99 (3):1461-76.

61. Rasch M, Logothetis NK, Kreiman G. From neurons to circuits: linear estimation of local field potentials. J Neurosci. 2009;29(44):13785-96.

62. WalterR WG, Cooper R, Aldridge VJ, McCallum WC, Winter AL. Contingent negative variation: an electric sign of sensorimotor association and expectancy in the human brain. Nature. 1964;203:380-4.

63. Blowers G, Ongley C, Shaw JC. The effect of reducing temporal expectancy on the contingent negative variation. Electroencephalogr Clin Neurophysiol. 1973;34(3):259-64.

64. Ruchkin DS, McCalley MG, Glaser EM. Event related potentials and time estimation. Psychophysiology. 1977;14(5):451-5.

65. Miniussi C, Wilding EL, Coull JT, Nobre AC. Orienting attention in time. Modulation of brain potentials. Br J Neurol. 1999;122(Pt 8):1507-18.

66. Macar F, Vidal F. The CNV peak: an index of decision making and temporal memory. Psychophysiology. 2003;40(6):950-4.

67. Pfeuty M, Ragot R, Pouthas V. Relationship between $\mathrm{CNV}$ and timing of an upcoming event. Neurosci Lett. 2005;382(1-2):106-11.

68. Praamstra P, Kourtis D, Kwok HF, Oostenveld R. Neurophysiology of implicit timing in serial choice reaction-time performance. J Neurosci. 2006;26 (20):5448-55.

69. Nauhaus I, Busse L, Carandini M, Ringach DL. Stimulus contrast modulates functional connectivity in visual cortex. Nat Neurosci. 2009;12(1):70-6.

70. Kopell N, Ermentrout GB, Whittington MA, Traub RD. Gamma rhythms and beta rhythms have different synchronization properties. Proc Natl Acad Sci U S A. 2000;97(4):1867-72.

71. Von Stein A, Sarnthein J. Different frequencies for different scales of cortical integration: from local gamma to long range alpha/theta synchronization. Int J Psychophysiol. 2000;38(3):301-13. 
72. Miller R. Theory of the normal waking EEG: from single neurones to waveforms in the alpha, beta and gamma frequency ranges. Int $\mathbf{J}$ Psychophysiol. 2007;64(1):18-23.

73. Whittington MA, Traub RD, Kopell N, Ermentrout B, Buhl EH. Inhibition-based rhythms: experimental and mathematical observations on network dynamics. Int J Psychophysiol. 2000;38(3):315-36.

74. Brunel N, Wang X-J. What determines the frequency of fast network oscillations with irregular neural discharges? I Synaptic dynamics and excitationinhibition balance. J Neurophysiol. 2003;90 (1):415-30

75. Jensen O, Goel P, Kopell N, Pohja M, Hari R, Ermentrout B. On the human sensorimotor-cortex beta rhythm: sources and modeling. Neuroimage. 2005;26(2):347-55.

76. Buzsáki G. Rhythms of the brain. New York: Oxford University Press; 2006.

77. Ray S, Maunsell JHR. Differences in gamma frequencies across visual cortex restrict their possible use in computation. Neuron. 2010;67(5):885-96.

78. Berger H. Über das Elektrenkephalogramm des Menschen. III. Arch Für Psychiatr Nervenkrankh. 1931;94:16-60.

79. Jasper H, Penfield W. Electrocorticograms in man: effect of voluntary movement upon the electrical activity of the precentral gyrus. Arch Für Psychiatr Z Neurol. 1949;183:163-74.

80. Murthy VN, Fetz EE. Coherent 25- to $35-\mathrm{Hz}$ oscillations in the sensorimotor cortex of awake behaving monkeys. Proc Natl Acad Sci U S A. 1992;89(12):5670-4.

81. Murthy VN, Fetz EE. Oscillatory activity in sensorimotor cortex of awake monkeys: synchronization of local field potentials and relation to behavior. J Neurophysiol. 1996;76(6):3949-67.

82. Roelfsema PR, Engel AK, König P, Singer W. Visuomotor integration is associated with zero time-lag synchronization among cortical areas. Nature. 1997;385(6612):157-61.

83. Brovelli A, Ding M, Ledberg A, Chen Y, Nakamura $\mathrm{R}$, Bressler SL. Beta oscillations in a large-scale sensorimotor cortical network: directional influences revealed by Granger causality. Proc Natl Acad Sci U S A. 2004;101(26):9849-54.

84. Courtemanche R, Fujii N, Graybiel AM. Synchronous, focally modulated beta-band oscillations characterize local field potential activity in the striatum of awake behaving monkeys. J Neurosci. 2003;23 (37):11741-52.

85. Courtemanche R, Lamarre Y. Local field potential oscillations in primate cerebellar cortex: synchronization with cerebral cortex during active and passive expectancy. J Neurophysiol. 2005;93(4):2039-52.

86. Kilavik BE, Zaepffel M, Brovelli A, MacKay WA, Riehle A. The ups and downs of $\beta$ oscillations in sensorimotor cortex. Exp Neurol. 2013;245:15-26.

87. Sanes JN, Donoghue JP. Oscillations in local field potentials of the primate motor cortex during voluntary movement. Proc Natl Acad Sci U S A. 1993;90(10):4470-4.

88. Donoghue JP, Sanes JN, Hatsopoulos NG, Gaál G. Neural discharge and local field potential oscillations in primate motor cortex during voluntary movements. J Neurophysiol. 1998;79(1):159-73.

89. Classen J, Gerloff C, Honda M, Hallett M. Integrative visuomotor behavior is associated with interregionally coherent oscillations in the human brain. J Neurophysiol. 1998;79(3):1567-73.

90. Saleh M, Reimer J, Penn R, Ojakangas CL, Hatsopoulos NG. Fast and slow oscillations in human primary motor cortex predict oncoming behaviorally relevant cues. Neuron. 2010;65(4):461-71.

91. Arnal LH. Predicting "When" using the motor system's beta-band oscillations. Front Hum Neurosci. 2012;6:225.

92. McIntosh GC, Brown SH, Rice RR, Thaut MH. Rhythmic auditory-motor facilitation of gait patterns in patients with Parkinson's disease. J Neurol Neurosurg Psychiatry. 1997;62(1):22-6.

93. Kilavik BE, Ponce-Alvarez A, Trachel R, Confais J, Takerkart S, Riehle A. Context-related frequency modulations of macaque motor cortical LFP beta oscillations. Cereb Cortex. 2012;22(9):2148-59.

94. Roux S, Mackay WA, Riehle A. The pre-movement component of motor cortical local field potentials reflects the level of expectancy. Behav Brain Res. 2006;169(2):335-51.

95. Hebb DO. The organization of behavior. New York: Wiley; 1949.

96. Aertsen A, Gerstein G, Johannesma P. From neuron to assembly: neuronal organization and stimulus representation. In: Palm G, Aertsen A, editors. Brain theory. Heidelberg: Springer; 1986. p. 7-24.

97. Gerstein GL, Bedenbaugh P, Aertsen MH. Neuronal assemblies. IEEE Trans Biomed Eng. 1989;36 (1):4-14.

98. Abeles M. Corticonics: neural circuits of the cerebral cortex. Cambridge: Cambridge University Press; 1991. 280 p.

99. Aertsen AHJ, Gerstein G. Dynamic aspects of neuronal cooperativity: fast stimulus-locked modulations of effective connectivity. In: Krüger J, editor. Neuronal cooperativity. Heidelberg: Springer; 1991. p. 52-67.

100. Kilavik BE, Roux S, Ponce-Alvarez A, Confais J, Grün S, Riehle A. Long-term modifications in motor cortical dynamics induced by intensive practice. J Neurosci. 2009;29(40):12653-63.

101. Rudolph M, Destexhe A. Tuning neocortical pyramidal neurons between integrators and coincidence detectors. J Comput Neurosci. 2003;14(3):239-51.

102. Grün S. Data-driven significance estimation for precise spike correlation. J Neurophysiol. 2009;101 (3):1126-40.

103. Weinrich M, Wise SP, Mauritz KH. A neurophysiological study of the premotor cortex in the rhesus monkey. Br J Neurol. 1984;107(Pt 2):385-414.

104. Riehle A. Visually induced signal-locked neuronal activity changes in precentral motor areas of the 
monkey: hierarchical progression of signal processing. Brain Res. 1991;540(1-2):131-7.

105. Riehle A, Requin J. Neuronal correlates of the specification of movement direction and force in four cortical areas of the monkey. Behav Brain Res. 1995;70(1):1-13.

106. Crammond DJ, Kalaska JF. Differential relation of discharge in primary motor cortex and premotor cortex to movements versus actively maintained postures during a reaching task. Exp Brain Res. 1996;108(1):45-61.

107. Cisek P, Kalaska JF. Neural correlates of reaching decisions in dorsal premotor cortex: specification of multiple direction choices and final selection of action. Neuron. 2005;45(5):801-14.

108. Kilavik BE, Confais J, Ponce-Alvarez A, Diesmann M, Riehle A. Evoked potentials in motor cortical local field potentials reflect task timing and behavioral performance. J Neurophysiol. 2010;104(5):2338-51.

109. Vaadia E, Benson DA, Hienz RD, Goldstein Jr MH. Unit study of monkey frontal cortex: active localization of auditory and of visual stimuli. J Neurophysiol. 1986;56(4):934-52.

110. Hoshi E, Tanji J. Functional specialization in dorsal and ventral premotor areas. Prog Brain Res. 2004; 143:507-11.

111. Hoshi E, Tanji J. Differential involvement of neurons in the dorsal and ventral premotor cortex during processing of visual signals for action planning. J Neurophysiol. 2006;95(6):3596-616.

112. Nakayama Y, Yamagata T, Tanji J, Hoshi E. Transformation of a virtual action plan into a motor plan in the premotor cortex. J Neurosci. 2008;28 (41):10287-97.

113. Yamagata T, Nakayama $Y$, Tanji J, Hoshi E. Processing of visual signals for direct specification of motor targets and for conceptual representation of action targets in the dorsal and ventral premotor cortex. J Neurophysiol. 2009;102(6):3280-94.

114. Yamagata T, Nakayama Y, Tanji J, Hoshi E. Distinct information representation and processing for goaldirected behavior in the dorsolateral and ventrolateral prefrontal cortex and the dorsal premotor cortex. J Neurosci. 2012;32(37):12934-49.

115. Riehle A, Requin J. Monkey primary motor and premotor cortex: single-cell activity related to prior information about direction and extent of an intended movement. J Neurophysiol. 1989;61(3):534-49.

116. Boussaoud D, Wise SP. Primate frontal cortex: effects of stimulus and movement. Exp Brain Res. 1993;95(1):28-40.

117. Boussaoud D, Wise SP. Primate frontal cortex: neuronal activity following attentional versus intentional cues. Exp Brain Res. 1993;95(1):15-27.

118. Kurata K. Information processing for motor control in primate premotor cortex. Behav Brain Res. 1994;61(2):135-42.

119. Wise SP, di Pellegrino G, Boussaoud D. The premotor cortex and nonstandard sensorimotor mapping. Can J Physiol Pharmacol. 1996;74 (4):469-82.

120. Riehle A, Kornblum S, Requin J. Neuronal correlates of sensorimotor association in stimulus-response compatibility. J Exp Psychol Hum Percept Perform. 1997;23(6):1708-26.

121. Shen L, Alexander GE. Neural correlates of a spatial sensory-to-motor transformation in primary motor cortex. J Neurophysiol. 1997;77(3):1171-94.

122. Shen L, Alexander GE. Preferential representation of instructed target location versus limb trajectory in dorsal premotor area. J Neurophysiol. 1997;77 (3):1195-212.

123. Bastian A, Schöner G, Riehle A. Preshaping and continuous evolution of motor cortical representations during movement preparation. Eur J Neurosci. 2003;18(7):2047-58.

124. Wise SP, Weinrich M, Mauritz KH. Motor aspects of cue-related neuronal activity in premotor cortex of the rhesus monkey. Brain Res. 1983;260(2):301-5.

125. Miller J, Riehle A, Requin J. Effects of preliminary perceptual output on neuronal activity of the primary motor cortex. J Exp Psychol Hum Percept Perform. 1992;18(4):1121-38.

126. Ledberg A, Bressler SL, Ding M, Coppola R, Nakamura R. Large-scale visuomotor integration in the cerebral cortex. Cereb Cortex. 2007;17(1):44-62.

127. Wise SP, Mauritz KH. Set-related neuronal activity in the premotor cortex of rhesus monkeys: effects of changes in motor set. Proc R Soc Lond B Biol Sci. 1985;223(1232):331-54.

128. Hikosaka O, Sakamoto M, Usui S. Functional properties of monkey caudate neurons. III. Activities related to expectation of target and reward. J Neurophysiol. 1989;61(4):814-32.

129. Lauwereyns J, Takikawa Y, Kawagoe R, Kobayashi S, Koizumi M, Coe B, et al. Feature-based anticipation of cues that predict reward in monkey caudate nucleus. Neuron. 2002;33(3):463-73.

130. Takikawa Y, Kawagoe R, Hikosaka O. Rewarddependent spatial selectivity of anticipatory activity in monkey caudate neurons. J Neurophysiol. 2002;87 (1):508-15.

131. Sakagami M, Niki H. Encoding of behavioral significance of visual stimuli by primate prefrontal neurons: relation to relevant task conditions. Exp Brain Res. 1994;97(3):423-36.

132. Rathelot J-A, Strick PL. Subdivisions of primary motor cortex based on cortico-motoneuronal cells. Proc Natl Acad Sci U S A. 2009;106(3):918-23.

133. Riehle A, Grammont F, MacKay WA. Cancellation of a planned movement in monkey motor cortex. Neuroreport. 2006;17(3):281-5.

134. Boussaoud D. Attention versus intention in the primate premotor cortex. Neuroimage. 2001;14(1 Pt 2): S40-5.

135. Lebedev MA, Wise SP. Tuning for the orientation of spatial attention in dorsal premotor cortex. Eur $\mathrm{J}$ Neurosci. 2001;13(5):1002-8. 
136. Coull JT, Nobre AC. Where and when to pay attention: the neural systems for directing attention to spatial locations and to time intervals as revealed by both PET and fMRI. J Neurosci. 1998;18(18):7426-35.

137. Coull JT, Frith CD, Büchel C, Nobre AC. Orienting attention in time: behavioural and neuroanatomical distinction between exogenous and endogenous shifts. Neuropsychologia. 2000;38(6):808-19.

138. Boulinguez P, Jaffard M, Granjon L, Benraiss A. Warning signals induce automatic EMG activations and proactive volitional inhibition: evidence from analysis of error distribution in simple RT. J Neurophysiol. 2008;99(3):1572-8.

139. Duque J, Ivry RB. Role of corticospinal suppression during motor preparation. Cereb Cortex. 2009;19 (9):2013-24.

140. Stuphorn V, Emeric EE. Proactive and reactive control by the medial frontal cortex. Front Neuroeng. 2012;5:9.

141. Moody SL, Wise SP. A model that accounts for activity prior to sensory inputs and responses during matching-to-sample tasks. J Cogn Neurosci. 2000;12 (3):429-48.

142. Cohen MR, Maunsell JHR. Attention improves performance primarily by reducing interneuronal correlations. Nat Neurosci. 2009;12(12):1594-600.
143. Sawaguchi T, Yamane I, Kubota K. Application of the GABA antagonist bicuculline to the premotor cortex reduces the ability to withhold reaching movements by well-trained monkeys in visually guided reaching task. J Neurophysiol. 1996;75(5): 2150-6.

144. Mirabella G, Pani P, Ferraina S. Neural correlates of cognitive control of reaching movements in the dorsal premotor cortex of rhesus monkeys. J Neurophysiol. 2011;106(3):1454-66.

145. Duque J, Labruna L, Verset S, Olivier E, Ivry RB. Dissociating the role of prefrontal and premotor cortices in controlling inhibitory mechanisms during motor preparation. J Neurosci. 2012;32(3):806-16.

146. Mauk MD, Buonomano DV. The neural basis of temporal processing. Annu Rev Neurosci. 2004;27:307-40.

147. Ivry RB, Schlerf JE. Dedicated and intrinsic models of time perception. Trends Cogn Sci. 2008;12(7): 273-80.

148. Merchant H, Harrington DL, Meck WH. Neural basis of the perception and estimation of time. Annu Rev Neurosci. 2013;36:313-36.

149. Grün S, Diesmann M, Aertsen A. Unitary events in multiple single-neuron spiking activity: II. Nonstationary data. Neural Comput. 2002;14(1):81-119. 